\title{
In situ carcinoma of pancreas diagnosed by EUS-FNA
}

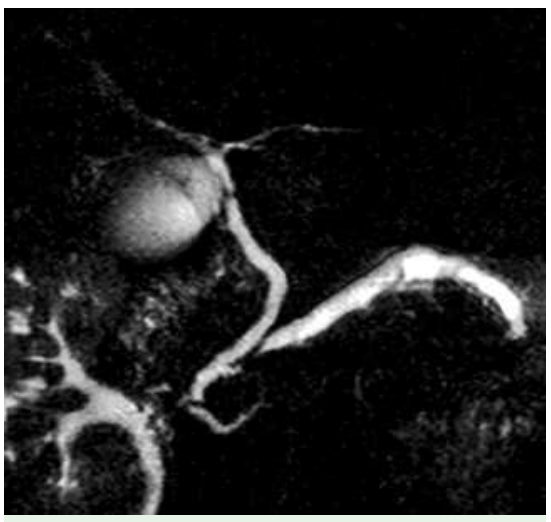

Fig. 1 Magnetic resonance cholangiopancreatography (MRCP) showing a stricture of the pancreatic duct and a dilatation of the distal duct.

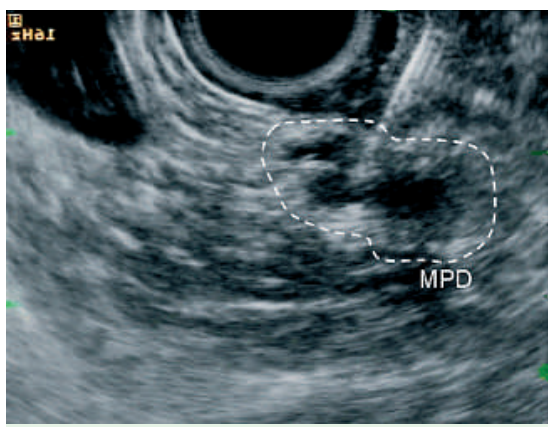

Fig. 2 EUS showing an echogenic structure $3 \mathrm{~mm}$ in diameter in the pancreatic duct (inside white dashed outline). This lesion is consistent with the ductal stenosis observed by MRCP. MPD: main pancreatic duct.

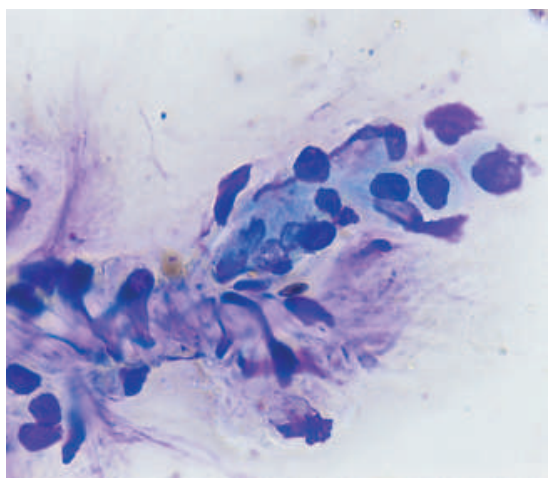

Fig. 3 Cytology of the samples obtained by EUS-FNA. A cluster of atypical cells is evident (Papanicolaou's stain, $\times 1250$ ).

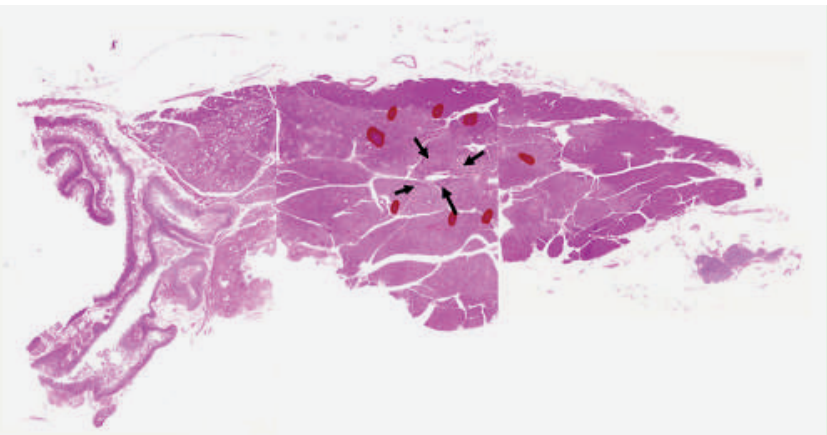

Fig. 4 Histopathological examination of surgical specimen reveals intraductally spreading carcinoma in the main duct of the pancreatic head. Red areas: inflammatory changes in the pancreas; arrows: pancreatic ducts (main and branch).

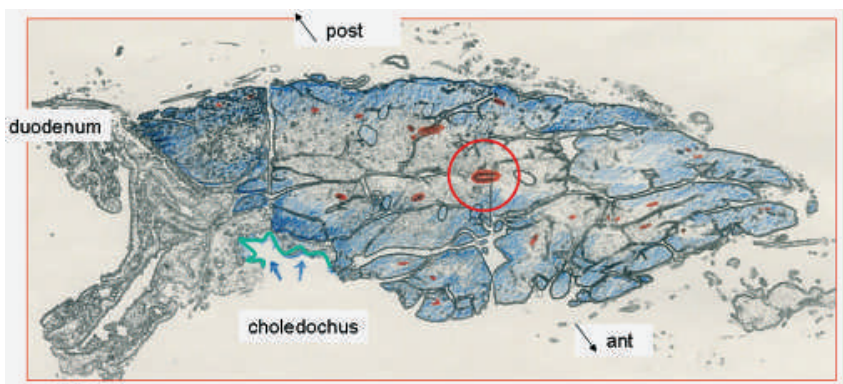

Fig. 5 Distribution of the carcinoma. Blue area: pancreas parenchyma; red areas: pancreatic ducts (main and branch); red circle: carcinoma in the main duct.

\section{Parenchyma of pancreas \\ Pancreatic duct (main and branch) \\ Stricture of main pancreatic duct with cancer inside}

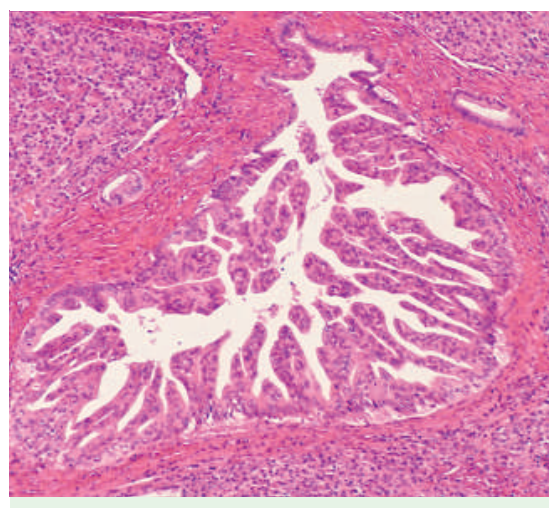

Fig. 6 Histopathological examination shows intraductally spreading carcinoma in the main duct of the pancreatic body $(H \& E, \times 250)$.

Endoscopic ultrasonography (EUS) is now a widely accepted modality for visualizing pancreatic lesions, and EUS-guided fine-needle aspiration (EUS-FNA) appears to be a safe and effective method for establishing the histological diagnosis of pancreatic tumors [1-3]. However, it is sometimes difficult to obtain samples from very small targets, especially tumors of $1 \mathrm{~cm}$ or less. We report a case in which EUS-FNA was useful for the diagnosis of in situ carcinoma of the pancreas. A 65year-old patient was admitted to our hospital because of dilatation of the main duct in the pancreatic body as seen on transabdominal ultrasonography. Serum carcinoembryonic antigen and carbohydrate antigen 19-9 levels were within normal limits. Computed tomography and magnetic resonance cholangiopancreatography (MRCP) revealed mild dilatation of the main pancreatic duct (๑ Fig. 1). During endoscopic retrograde pancreatography, deep cannulation proved impossible due to severe stricture of the pancreatic duct, and therefore no adequate sample of pancreatic juice could obtained. EUS showed an echogenic structure $3 \mathrm{~mm}$ in diameter and $5 \mathrm{~mm}$ in length in the stenotic duct of the pancreas (- Fig. 2). EUS-FNA was performed for a histological diagnosis of the echogenic structure in the pancreatic duct. The cytological examination demonstrated clusters of atypical cells consistent with adenocarcinoma ( Fig. 3). The histopathological examination of the pancreas achieved by pancreatic duodenectomy 
showed an intraductally spreading carcinoma that was $8 \mathrm{~mm}$ in maximum length, $3 \mathrm{~mm}$ in diameter, in the main duct of the pancreatic body. The histopathological diagnosis was in situ carcinoma of the pancreas ( Fig. 4-6).

Endoscopy_UCTN_Code_CCL_1AF_2AZ_3AB

\section{H. Sakamoto ${ }^{1}$, M. Kitano ${ }^{1}$, K. Dote ${ }^{2}$}

T. Tchikugo ${ }^{2,3}$, Y. Takeyama ${ }^{3}$, M. Kudo

1 Department of Gastroenterology and Hepatology, Kinki University School of Medicine, Osaka-Sayama, Japan

2 Department of Pathology, Kinki University School of Medicine, Osaka-Sayama, Japan

3 Department of Surgery, Kinki University School of Medicine, Osaka-Sayama,

Japan

\section{References}

1 Vilmann P, Jacobsen GK, Henriksen FW et al. Endoscopic ultrasonography with guided fine needle aspiration biopsy in pancreatic disease. Gastrointest Endosc 1992; 38 $172-173$

2 O'Toole D, Palazzo L, Arotçarena R et al. Assessment of complication of EUS-guided fine-needle aspiration. Gastrointest Endosc 2001; 53: 470-474

3 Bhutani MS, Hawes RH, Baron PL et al. Endoscopic ultrasound guided fine needle aspiration of malignant pancreatic lesions. Endoscopy 1997; 29: 854-858
Bibliography

DOI $10.1055 / \mathrm{s}-2007-966708$

Endoscopy 2008; 40: E15-E16

(c) Georg Thieme Verlag KG Stuttgart · New York . ISSN 0013-726X

\section{Corresponding author}

\section{Kitano, MD, PhD}

Division of Gastroenterology and Hepatology Department of Internal Medicine Kinki University School of Medicine 377-2, Ohno-Higashi

Osaka-Sayama, 589-8511

Japan

Fax: +81-72-3672880

kitano@med.kindai.ac.jp 